\title{
ZOOGEOGRAPHICAL REGIONALIZATION OF THE SERBIA ACCORDING TO THE AFFINITY OF LOCAL FAUNAS OF THE SKIPPERS AND BUTTERFLIES (LEPIDOPTERA: HESPERIOIDEA \& PAPILIONOIDEA)
}

\author{
Predrag Jakšić ${ }^{1 *}$ \\ ${ }^{1}$ Faculty of Sciences and Mathematics, University of Niš, Niš, Serbia.
}

\begin{abstract}
Integrated list of skippers and butterflies of Serbia is shown. We also conducted faunal analyses of established species. Based on previous research, some regions have been identified as center of certain faunal groups. Degree of similarity of selected areas is established by Jaccard index

method. By Cluster analyses has identified links between isolated areas. Specific faunal elements of certain areas have been separated - they are diferencial species. Summerazing the obtain results, zoogeographical map of the skippers and butterflies of Serbia is done.
\end{abstract}

Key words: butterflies, faunal elements, zogeography, Serbia

\section{INTRODUCTION}

According to modern concept, diversity center of Lepidoptera in Palaearctic area is Western China. This area was settled by ancestor line from Gondvana (Varga, 2002). That is where migration and diversification began. Thanks to that greatest number of European skippers and butterflies belong to Palaearctic species group. East Asia species using the northern route (closed forest habitat) are indicated as Arboreal. East Asia species using the southern route (open habitats) are indicated as Oreal. Balkan Peninsula has been inhabited by both groups. That process has been repeated on several occasions from several directions. The success of migration depended on local environmental factors. Jagged territory enabled survival of species of different affinities. Thanks to that, particular territory has specific assemblage of species. That is, we talk about "faunal elements"

The main zoogeographical task is to establish faunal and areal elements of certain groups. Based on that, further on, zoogeographical data chart can be done.

The skippers and butterfliy faunal element research and definition has a long tradition. The first significant contribution was given by (Pagenstecher, 1909), than (Hormuzaki, 1929, 1930), (Rebel, 1932-33) as the leading expert of that time was first to conduct faunal analyses of skippers and butterflies of Balkan Peninsula. After Rebel several authors (see references: Annotated Bibliography) have contributed faunal elements definition. In this paper we addopted the system given by (Kudrna et al., 2015).

Vegetation and phytogeographic chart was first done by (Adamović, 1907), followed by Fukarek and (Jovanović, 1983), (Gajić, 1984) and (Stevanović et al., 1995).

The first zoogeographic chart of Serbia was done by (Kobelt, 1904) based on Mollusca. He singled out three provinces: Carpathian-Transilvanian, North Balkan as well as Albanian-Macedonian. Some times later (Pavlović, 1912), based on snail studies of Serbia in that time, singled out five provinces. The first zoogeographic chart of entire contemporary Serbian territory, was done by (Hadži, 1931) based on cryptobiotic species study. Finaly, (Matvejev, 1968) did zoogeographic chart of Serbia based on birds distribution.

Having that in mind we can come up with an assumption (as antithesis) that Serbia has skippers and butterflies with heterogenus composition and historically conditioned disposition and in a compliance with current ecological condition. Accordingly, Serbian territory in a sense of zoogeography, is not homogenus but with clearly distinguished areas.

\section{MATERIALS AND METHODS}

In order to answer these questions eleven provinces on the territory of Serbia has been chosen. 
Faunistic data were taken from the existing literature (Tab. 1).

(Jaccard's, 1902) index was used to present the degree of dissimilarity between zoogeographic regions: $\mathrm{R}=100 \mathrm{C} / \mathrm{A}+\mathrm{B}-\mathrm{C}$

where:

$\mathrm{A}=$ number of species in the richest fauna;

$\mathrm{B}=$ number of species in the poorest fauna;

$\mathrm{C}=$ number of species common to both faunas.

For agglomerate clustering, UPGMA method were used (Sokal and Rohlf, 1995). Estimations were done using the program FLORA (Karadžić and Marinković, 2009).

\section{RESULTS}

Based on previous studies of butterfly fauna of Serbia (Tab. 1) list of established species has been done. Distribution of species is shown broken into 11 areas, that are set aside on basis of previous findings. Faunistic affiliation of each species is added, according to (Kudrna et al., 2015).

Table 1. List of provinces and previous papers on distribution of butterflies in Serbia.

\begin{tabular}{|l|l|l|}
\hline No. & Provinces & Data for Butterflies (References) \\
\hline 1 & Pannonicum & $\begin{array}{l}\text { Andjus, 2008; Jakšić i Nahirnić, 2011; Jakšić et al., 2008; Lorković und Siladjev, 1982; } \\
\text { Popović et al., 2014. }\end{array}$ \\
\hline 2 & Dacicum & Andjus, 2008; Zečević, 2002. \\
\hline 3 & Carpathicum & Gradojević,1930-31; Jakšić, 2006.; Zečević, 2002; Živojinović,1950. \\
\hline 4 & Moesicum & Jakšić, 2011; Popović i Đurić, 2014; Swaay et al., 2007; Tuleschkow,1932. \\
\hline 5 & W-Rhodope & Jakšić, 2003; Nahirnić, 2011; Swaay et al., 2007. \\
\hline 6 & Serbicum & Gradojević,1930-31; Jakšić, 2015. \\
\hline 7 & Dinaricum (Illyricum) & $\begin{array}{l}\text { Dodok, 2003; Đurić \& Franeta, 2011; Jakšić, 2011; Jakšić i Nahirnić, 2014.; Nahirnić et } \\
\text { al. 2015. }\end{array}$ \\
\hline 8 & Bertiscum & Jakšić, 2003- \\
\hline 9 & Scardicum & Jakšić, 1998. \\
\hline 10. & $\begin{array}{l}\text { 10a. Sub-Aegean } \\
10 b . \text { Sub-Adriaticum }\end{array}$ & $\begin{array}{l}\text { Jakšić, 1986; Jakšić \& Ristić, 1999; Kogovšek et al., 2012; Popović et al., 2014; Swaay et } \\
\text { al., 2007. }\end{array}$ \\
\hline 11. & Ponišavlje & Popović i Đurić, 2014; Todorova, und Petkoff, P., 1915 \\
\hline
\end{tabular}

Table. 2. List of butterfly species recorded in Serbia. Faunal elements according to (Kudrna et al., 2015).

\begin{tabular}{|c|c|c|c|c|c|c|c|c|c|c|c|c|}
\hline & & 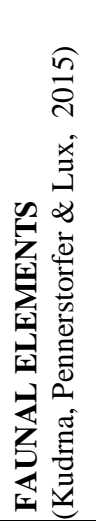 & 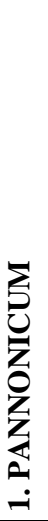 & 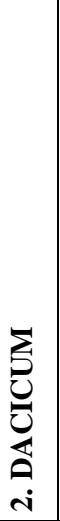 & 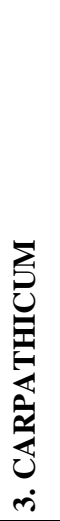 & 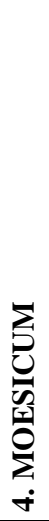 & 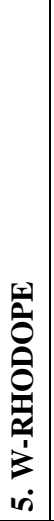 & 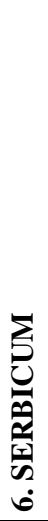 & 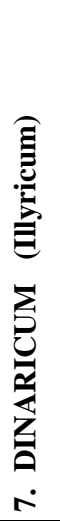 & 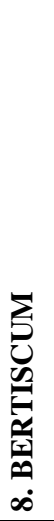 & 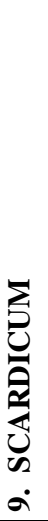 & 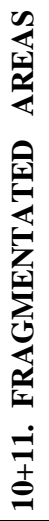 \\
\hline & LEPIDOPTERA & & & & & & & & & & & \\
\hline & HESPERIOIDEA & & & & & & & & & & & \\
\hline & HESPERIIDAE & & & & & & & & & & & \\
\hline 1 & Erynnis tages (Linnaeus, 1758) & ES & 1 & 1 & 1 & 1 & 1 & 1 & 1 & 1 & 1 & 1 \\
\hline 2 & Carcharodus alceae (Esper, 1780) & Med & 1 & 1 & 1 & 1 & 1 & 1 & 1 & 1 & 1 & 1 \\
\hline 3 & Carcharodus lavatherae (Esper, 1783) & EM & 1 & 1 & 0 & 1 & 0 & 1 & 1 & 0 & 0 & 1 \\
\hline 4 & Carcharodus flocciferus (Zeller, 1847) & EO & 1 & 0 & 1 & 1 & 0 & 1 & 1 & 0 & 1 & 0 \\
\hline 5 & Spialia phlomidis (Herrich-Schäffer, 1845) & EO & 0 & 0 & 0 & 0 & 0 & 0 & 1 & 0 & 0 & 1 \\
\hline 6 & Spialia orbifer (Hübner, 1823) & EO & 1 & 1 & 0 & 1 & 1 & 1 & 1 & 1 & 1 & 0 \\
\hline 7 & Syrichtus proto (Ochsenheimer, 1808) & EO & 0 & 0 & 0 & 0 & 0 & 0 & 0 & 0 & 1 & 0 \\
\hline 8 & Syrichtus cribrellum (Eversmann, 1841) & ES & 0 & 0 & 0 & 0 & 0 & 0 & 0 & 0 & 0 & 1 \\
\hline 9 & Pyrgus carthami (Hübner, 1813) & EO & 1 & 0 & 1 & 1 & 1 & 1 & 1 & 0 & 0 & 0 \\
\hline 10 & Pyrgus sidae (Esper, 1782) & EO & 0 & 0 & 0 & 1 & 1 & 1 & 0 & 0 & 1 & 0 \\
\hline 11 & Pyrgus andromedae (Wallengren, 1853) & $\mathrm{BM}$ & 0 & 0 & 0 & 0 & 0 & 0 & 0 & 0 & 1 & 0 \\
\hline 12 & Pyrgus malvae (Linnaeus, 1758) & ES & 1 & 1 & 1 & 1 & 1 & 1 & 1 & 1 & 1 & 1 \\
\hline
\end{tabular}

\section{Biology}




\begin{tabular}{|c|c|c|c|c|c|c|c|c|c|c|c|c|}
\hline 13 & Pyrgus serratulae (Rambur, 1840) & $\mathrm{ES}$ & 1 & 0 & 0 & 1 & 0 & 1 & 1 & 1 & 0 & 0 \\
\hline 14 & Pyrgus cinarae (Rambur, 1840 ) & $\mathrm{EO}$ & 0 & 0 & 0 & 0 & 0 & 0 & 0 & 0 & 0 & 1 \\
\hline 15 & Pyrgus armoricanus (Oberthur, 1910) & EO & 1 & 1 & 1 & 1 & 0 & 1 & 1 & 1 & 1 & 0 \\
\hline 16 & Pyrgus alveus (Hübner, 1803) & ES & 1 & 0 & 1 & 1 & 0 & 1 & 1 & 1 & 1 & 0 \\
\hline 17 & Pyrgus trenbevicensis (Warren, 1926) & ES & 0 & 0 & 1 & 0 & 0 & 0 & 1 & 0 & 0 & 0 \\
\hline 18 & Heteropterus morpheus (Pallas, 1771) & ES & 0 & 0 & 1 & 0 & 0 & 1 & 1 & 0 & 0 & 0 \\
\hline 19 & Carterocephalus palaemon (Pallas, 1771) & Hol & 0 & 0 & 0 & 1 & 0 & 1 & 1 & 1 & 0 & 1 \\
\hline 20 & Thymelicus lineola (Ochsenheimer, 1806) & Hol & 0 & 0 & 1 & 1 & 1 & 1 & 1 & 1 & 1 & 1 \\
\hline 21 & Thymelicus sylvestris (Poda, 1761) & EO & 1 & 0 & 1 & 1 & 1 & 1 & 1 & 1 & 0 & 0 \\
\hline 22 & Thymelicus acteon (Rottemburg, 1775) & $\mathrm{EO}$ & 0 & 0 & 1 & 1 & 1 & 1 & 0 & 0 & 0 & 0 \\
\hline 23 & Hesperia comma (Linnaeus, 1758) & Hol & 1 & 0 & 1 & 1 & 0 & 1 & 1 & 1 & 1 & 0 \\
\hline \multirow[t]{3}{*}{24} & Ochlodes sylvanus (Esper, 1777) & ES & 1 & 1 & 1 & 1 & 1 & 1 & 1 & 1 & 1 & 1 \\
\hline & PAPILIONOIDEA & & & & & & & & & & & \\
\hline & PAPILIONIDAE & & & & & & & & & & & \\
\hline 25 & Zerynthia polyxena (Schiffermüller, 1775) & EO & 1 & 0 & 0 & 1 & 1 & 1 & 1 & 0 & 1 & 0 \\
\hline 26 & Zerynthia cerisy (Godart, 1822) & EO & 0 & 1 & 1 & 1 & 1 & 0 & 0 & 0 & 0 & 1 \\
\hline 27 & Parnassius mnemosyne (Linnaeus, 1758) & EO & 1 & 0 & 1 & 1 & 1 & 1 & 1 & 1 & 1 & 1 \\
\hline 28 & Parnassius apollo (Linnaeus, 1758) & $\mathrm{ES}$ & 0 & 0 & 1 & 1 & 0 & 0 & 1 & 1 & 1 & 0 \\
\hline 29 & Iphiclides podalirius (Linnaeus, 1758) & $\mathrm{ES}$ & 1 & 1 & 1 & 1 & 1 & 1 & 1 & 1 & 1 & 1 \\
\hline \multirow[t]{2}{*}{30} & Papilio machaon (Linnaeus, 1758) & $\mathrm{ES}$ & 1 & 1 & 1 & 1 & 1 & 1 & 1 & 1 & 1 & 1 \\
\hline & PIERIDAE & & & & & & & & & & & \\
\hline 31 & Leptidea sinapis (Linnaeus, 1758) complex & ES & 1 & 1 & 1 & 1 & 1 & 1 & 1 & 1 & 1 & 1 \\
\hline 32 & $\begin{array}{l}\text { Leptidea reali (Reissinger, 1989) - juvernica } \\
\text { Williams, } 1946 \text { complex }\end{array}$ & ES & 0 & 0 & 1 & 1 & 1 & 1 & 1 & 1 & 1 & 1 \\
\hline 33 & Leptidea duponcheli (Staudinger, 1871) & EO & 0 & 0 & 0 & 0 & 1 & 1 & 0 & 1 & 0 & 1 \\
\hline 34 & Leptidea morsei (Fenton, 1882) & ES & 1 & 0 & 0 & 0 & 0 & 0 & 0 & 0 & 0 & 0 \\
\hline 35 & Anthocharis cardamines (Linnaeus, 1758) & ES & 1 & 1 & 1 & 1 & 1 & 1 & 1 & 1 & 1 & 1 \\
\hline 36 & Anthocharis gruneri (Herrich-Schäffer, 1851) & EO & 0 & 0 & 0 & 0 & 0 & 0 & 0 & 0 & 0 & 1 \\
\hline 37 & Euchloe ausonia (Hübner, 1806) complex & Med & 1 & 1 & 0 & 0 & 0 & 0 & 0 & 0 & 0 & 1 \\
\hline 38 & Aporia crataegi (Linnaeus, 1758) & ES & 1 & 1 & 1 & 1 & 1 & 1 & 1 & 0 & 1 & 1 \\
\hline 39 & Pieris brassicae (Linnaeus, 1758) & $\mathrm{ES}$ & 1 & 1 & 1 & 1 & 1 & 1 & 1 & 1 & 1 & 1 \\
\hline 40 & Pieris mannii (Mayer, 1851) & EO & 0 & 1 & 1 & 1 & 0 & 0 & 1 & 1 & 1 & 0 \\
\hline 41 & Pieris rapae (Linnaeus, 1758) & Hol & 1 & 1 & 1 & 1 & 1 & 1 & 1 & 1 & 1 & 1 \\
\hline 42 & Pieris ergane (Geyer, 1828) & EO & 0 & 0 & 0 & 1 & 0 & 1 & 1 & 1 & 1 & 0 \\
\hline 43 & Pieris napi (Linnaeus, 1758) & ES & 1 & 1 & 1 & 1 & 1 & 1 & 1 & 1 & 1 & 1 \\
\hline 44 & Pieris balcana (Lorković, 1968) & ES & 0 & 0 & 0 & 0 & 0 & 1 & 1 & 1 & 1 & 0 \\
\hline 45 & Pontia edusa (Fabricius, 1777) & ES & 1 & 0 & 1 & 1 & 0 & 1 & 1 & 1 & 1 & 0 \\
\hline 46 & Colias erate (Esper, 1803$)$ & $\mathrm{ES}$ & 1 & 1 & 1 & 0 & 1 & 0 & 0 & 0 & 0 & 1 \\
\hline 47 & Colias crocea (Fourcroy, 1785) & EO & 1 & 1 & 1 & 1 & 1 & 1 & 1 & 1 & 1 & 1 \\
\hline 48 & Colias myrmidone (Esper, 1781) & EO & 1 & 1 & 1 & 0 & 0 & 0 & 0 & 0 & 0 & 0 \\
\hline 49 & Colias balcanica (Rebel, 1903) & Mon & 0 & 0 & 0 & 0 & 0 & 0 & 1 & 0 & 0 & 0 \\
\hline 50 & Colias hyale (Linnaeus, 1758) & ES & 1 & 1 & 1 & 1 & 1 & 1 & 1 & 1 & 1 & 1 \\
\hline 51 & Colias alfacariensis (Ribbe, 1905 ) & EO & 1 & 1 & 1 & 1 & 1 & 1 & 1 & 1 & 1 & 1 \\
\hline \multirow[t]{2}{*}{52} & Gonepteryx rhamni (Linnaeus, 1758) & ES & 1 & 1 & 1 & 1 & 1 & 1 & 1 & 1 & 1 & 1 \\
\hline & RIODINIDAE & & & & & & & & & & & \\
\hline \multirow[t]{2}{*}{53} & Hamearis lucina (Linnaeus, 1758) & EM & 1 & 1 & 1 & 1 & 1 & 1 & 1 & 1 & 1 & 1 \\
\hline & LYCAENIDAE & & & & & & & & & & & \\
\hline 54 & Lycaena phlaeas (Linnaeus, 1761) & Hol & 1 & 1 & 1 & 1 & 1 & 1 & 1 & 1 & 1 & 1 \\
\hline 55 & Lycaena helle (Schiffermüller, 1775) & ES & 0 & 0 & 0 & 1 & o & 0 & 0 & 0 & 0 & 0 \\
\hline 56 & Lycaena dispar (Haworth, 1802) & $\mathrm{ES}$ & 1 & 1 & 1 & 1 & 1 & 1 & 1 & 1 & 1 & 1 \\
\hline 57 & Lycaena virgaureae (Linnaeus, 1758) & ES & 0 & 0 & 1 & 1 & 1 & 0 & 1 & 1 & 1 & 1 \\
\hline 58 & Lycaena tityrus (Poda, 1761$)$ & ES & 1 & 1 & 1 & 1 & 1 & 1 & 1 & 1 & 1 & 1 \\
\hline 59 & Lycaena alciphron (Rottemburg, 1775) & EO & 0 & 1 & 1 & 1 & 1 & 1 & 1 & 1 & 1 & 0 \\
\hline 60 & Lycaena hippothoe (Linnaeus, 1760) & ES & 0 & 0 & 0 & 0 & 0 & 0 & 1 & 0 & 0 & 0 \\
\hline 61 & Lycaena candens (Herrich-Schäffer, 1844) & Mon & 0 & 0 & 1 & 1 & 0 & 0 & 1 & 1 & 1 & 0 \\
\hline 62 & Lycaena thersamon (Esper, 1784) & EO & 0 & 0 & 1 & 1 & 1 & 1 & 1 & 1 & 1 & 1 \\
\hline 63 & Thecla betulae (Linnaeus, 1758) & ES & 0 & 1 & 0 & 1 & 1 & 1 & 1 & 0 & 1 & 0 \\
\hline 64 & Favonius quercus (Linnaeus, 1758) & EO & 1 & 1 & 1 & 1 & 1 & 1 & 1 & 1 & 1 & 0 \\
\hline 65 & Callophrys rubi (Linnaeus, 1758) & ES & 1 & 1 & 1 & 1 & 1 & 1 & 1 & 1 & 1 & 1 \\
\hline 66 & Satyrium w-album (Knoch, 1782) & ES & 1 & 1 & 0 & 1 & 0 & 1 & 1 & 1 & 1 & 0 \\
\hline
\end{tabular}

\section{Biology}




\begin{tabular}{|c|c|c|c|c|c|c|c|c|c|c|c|c|}
\hline 67 & Satyrium pruni (Linnaeus, 1758) & ES & 1 & 1 & 0 & 1 & 0 & 1 & 1 & 0 & 1 & 0 \\
\hline 68 & Satyrium spini (Fabricius, 1787) & EO & 1 & 1 & 1 & 1 & 1 & 1 & 1 & 1 & 1 & 0 \\
\hline 69 & Satyrium ilicis (Esper, 1779) & EO & 0 & 1 & 0 & 1 & 1 & 1 & 1 & 1 & 1 & 0 \\
\hline 70 & Satyrium acaciae (Fabricius, 1787) & EO & 1 & 1 & 1 & 1 & 1 & 1 & 1 & 1 & 0 & 1 \\
\hline 71 & Lampides boeticus (Linnaeus, 1767) & Tro & 0 & 0 & 0 & 1 & 0 & 1 & 1 & 1 & 0 & 0 \\
\hline 72 & Leptotes pirithous (Linnaeus, 1767) & Tro & 0 & 1 & 0 & 1 & 1 & 1 & 1 & 0 & 1 & 0 \\
\hline 73 & Cupido minimus (Fuessly, 1775) & ES & 1 & 0 & 1 & 1 & 1 & 0 & 1 & 1 & 1 & 1 \\
\hline 74 & Cupido osiris (Meigen, 1829) & EO & 0 & 0 & 1 & 1 & 0 & 1 & 1 & 0 & 1 & 1 \\
\hline 75 & Cupido argiades (Pallas, 1771) & Hol & 1 & 0 & 1 & 1 & 1 & 1 & 1 & 0 & 0 & 1 \\
\hline 76 & Cupido decolorata (Staudinger, 1886) & EM & 0 & 0 & 1 & 1 & 0 & 1 & 1 & 0 & 1 & 1 \\
\hline 77 & Cupido alcetas (Hoffmannsegg, 1803) & ES & 1 & 1 & 1 & 1 & 0 & 1 & 1 & 0 & 1 & 1 \\
\hline 78 & Celastrina argiolus (Linnaeus, 1758) & ES & 1 & 1 & 1 & 1 & 1 & 1 & 1 & 1 & 1 & 1 \\
\hline 79 & Pseudophilotes vicrama (Moore, 1865$)$ & EO & 0 & 0 & 0 & 1 & 1 & 1 & 1 & 0 & 1 & 1 \\
\hline 80 & Pseudophilotes bavius (Eversmann, 1832) & EM & 0 & 0 & 0 & 0 & 0 & 0 & 0 & 1 & 1 & 0 \\
\hline 81 & Scolitantides orion (Pallas, 1771) & ES & 0 & 1 & 1 & 1 & 1 & 0 & 1 & 1 & 1 & 1 \\
\hline 82 & Glaucopsyche alexis (Poda, 1761) & ES & 1 & 1 & 0 & 1 & 0 & 1 & 1 & 1 & 1 & 1 \\
\hline 83 & Iolana iolas (Ochsenheimer, 1816) & EO & 0 & 0 & 0 & 0 & 1 & 1 & 1 & 0 & 1 & 0 \\
\hline 84 & Phengaris arion (Linnaeus, 1758) & ES & 0 & 1 & 1 & 1 & 0 & 1 & 1 & 1 & 1 & 1 \\
\hline 85 & Phengaris teleius (Bergsträsser, 1779) & ES & 1 & 0 & 0 & 0 & 0 & 0 & 0 & 0 & 0 & 0 \\
\hline 86 & Phengaris alcon (Schiffermüller, 1775) & ES & 0 & 0 & 0 & 1 & 1 & 1 & 1 & 1 & 1 & 0 \\
\hline 87 & Kretania pylaon (Fischer, 1832) complex & ES & 1 & 0 & 1 & 1 & 0 & 1 & 1 & 0 & 0 & 0 \\
\hline 88 & Plebeius argus (Linnaeus, 1758) & ES & 1 & 1 & 1 & 1 & 1 & 1 & 1 & 1 & 1 & 1 \\
\hline 89 & Plebeius idas (Linnaeus, 1760) & Hol & 1 & 1 & 1 & 1 & 1 & 1 & 1 & 1 & 1 & 1 \\
\hline 90 & Plebeius argyrognomon (Bergsträsser, 1779) & ES & 1 & 0 & 0 & 1 & 0 & 1 & 1 & 1 & 1 & 1 \\
\hline 91 & Agriades optilete (Knoch, 1781$)$ & $\mathrm{BM}$ & 0 & 0 & 0 & 0 & 0 & 0 & 0 & 0 & 1 & 0 \\
\hline 92 & Eumedonia eumedon (Esper, 1780) & ES & 0 & 0 & 0 & 1 & 0 & 1 & 1 & 0 & 1 & 0 \\
\hline 93 & Aricia agestis (Schiffermüller, 1775) & ES & 1 & 1 & 1 & 1 & 1 & 1 & 1 & 1 & 1 & 1 \\
\hline 94 & Aricia artaxerxes (Fabricius, 1793) & $\mathrm{BM}$ & 0 & 1 & 0 & 1 & 1 & 1 & 1 & 1 & 1 & 0 \\
\hline 95 & Aricia anteros (Freyer, 1838) & Mon & 0 & 0 & 1 & 1 & 0 & 0 & 1 & 1 & 1 & 1 \\
\hline 96 & Cyaniris semiargus (Rottemburg, 1775) & ES & 1 & 1 & 1 & 1 & 1 & 1 & 1 & 1 & 1 & 1 \\
\hline 97 & Polyommatus escheri (Hübner, 1823) & EM & 0 & 0 & 0 & 0 & 0 & 0 & 1 & 0 & 1 & 0 \\
\hline 98 & Polyommatus dorylas (Schiffermüller, 1775) & $\mathrm{EO}$ & 0 & 0 & 1 & 1 & 1 & 0 & 1 & 1 & 1 & 0 \\
\hline 99 & $\begin{array}{l}\text { Polyommatus icarius (Esper, 1789) (syn. } \\
\text { amandus) }\end{array}$ & ES & 1 & 0 & 0 & 1 & 1 & 1 & 1 & 0 & 1 & 1 \\
\hline 100 & Polyommatus thersites (Cantener, 1834) & $\mathrm{ES}$ & 0 & 0 & 1 & 1 & 1 & 1 & 0 & 1 & 1 & 0 \\
\hline 101 & Polyommatus icarus (Rottemburg, 1775) & ES & 1 & 1 & 1 & 1 & 1 & 1 & 1 & 1 & 1 & 1 \\
\hline 102 & Polyommatus eros (Ochsenheimer, 1808) & ES & 0 & 0 & 1 & 1 & 0 & 0 & 1 & 0 & 1 & 0 \\
\hline 103 & Polyommatus daphnis (Schiffermüller, 1775) & EO & 0 & 1 & 1 & 1 & 1 & 1 & 1 & 1 & 1 & 1 \\
\hline 104 & Polyommatus bellargus (Rottemburg, 1775) & EO & 1 & 1 & 1 & 1 & 1 & 1 & 1 & 1 & 1 & 0 \\
\hline 105 & Polyommatus coridon (Poda, 1761$)$ & EO & 1 & 1 & 1 & 1 & 0 & 1 & 1 & 1 & 1 & 1 \\
\hline 106 & Polyommatus admetus (Esper, 1783) & EO & 0 & 0 & 0 & 1 & 1 & 1 & 0 & 0 & 1 & 0 \\
\hline 107 & Polyommatus ripartii (Freyer, 1830) & EO & 0 & 0 & 0 & 1 & 0 & 1 & 0 & 1 & 0 & 0 \\
\hline 108 & Polyommatus damon (Schiffermüller, 1775) & ES & 0 & 0 & 0 & 0 & 0 & 0 & 0 & 1 & 1 & 1 \\
\hline & NYMPHALIDAE & & & & & & & & & & & \\
\hline 109 & Libythea celtis (Laicharting, 1782) & EO & 1 & 1 & 0 & 0 & 0 & 0 & 1 & 1 & 1 & 1 \\
\hline 110 & Argynnis paphia (Linnaeus, 1758) & ES & 1 & 1 & 1 & 1 & 1 & 1 & 1 & 1 & 1 & 1 \\
\hline 111 & Argynnis pandora (Schiffermüller, 1775) & EO & 1 & 1 & 1 & 1 & 0 & 1 & 1 & 1 & 1 & 0 \\
\hline 112 & Argynnis aglaja (Linnaeus, 1758) & ES & 1 & 1 & 1 & 1 & 1 & 1 & 1 & 1 & 1 & 0 \\
\hline 113 & Argynnis adippe ([Schiffermüller], 1775) & ES & 0 & 1 & 1 & 1 & 1 & 1 & 1 & 1 & 1 & 0 \\
\hline 114 & Argynnis niobe (Linnaeus, 1758) & ES & 0 & 1 & 1 & 1 & 1 & 1 & 1 & 1 & 1 & 0 \\
\hline 115 & Issoria lathonia (Linnaeus, 1758) & ES & 1 & 1 & 1 & 1 & 1 & 1 & 1 & 1 & 1 & 1 \\
\hline 116 & Brenthis ino (Rottemburg, 1775) & ES & 0 & 0 & 0 & 1 & 0 & 0 & 0 & 0 & 0 & 0 \\
\hline 117 & Brenthis daphne (Bergsträsser, 1780) & ES & 1 & 1 & 1 & 1 & 1 & 1 & 1 & 1 & 1 & 1 \\
\hline 118 & Brenthis hecate (Schiffermüller, 1775) & ES & 0 & 1 & 1 & 1 & 1 & 1 & 1 & 0 & 1 & 0 \\
\hline 119 & Boloria eunomia (Esper, 1799) & Hol & 0 & 0 & 1 & 1 & 1 & 0 & 0 & 0 & 0 & 0 \\
\hline 120 & Boloria euphrosyne (Linnaeus, 1758) & ES & 0 & 0 & 1 & 1 & 1 & 1 & 1 & 1 & 1 & 1 \\
\hline 121 & Boloria titania (Esper, 1793) & Hol & 0 & 0 & 0 & 0 & 0 & 0 & 1 & 1 & 1 & 0 \\
\hline 122 & Boloria selene (Schiffermüller, 1775) & Hol & 0 & 0 & 1 & 1 & 0 & 0 & 1 & 0 & 0 & 0 \\
\hline 123 & Boloria dia (Linnaeus, 1767) & ES & 1 & 1 & 1 & 1 & 1 & 1 & 1 & 1 & 1 & 1 \\
\hline 124 & Boloria pales (Schiffermüller, 1775) & Mon & 0 & 0 & 0 & 0 & 0 & 0 & 0 & 1 & 1 & 0 \\
\hline 125 & Boloria graeca (Staudinger, 1870) & Mon & 0 & 0 & 0 & 0 & 0 & 0 & 0 & 1 & 1 & 0 \\
\hline 126 & Vanessa atalanta (Linnaeus, 1758) & Hol & 1 & 1 & 1 & 1 & 1 & 1 & 1 & 1 & 1 & 1 \\
\hline
\end{tabular}

\section{Biology}




\begin{tabular}{|c|c|c|c|c|c|c|c|c|c|c|c|c|}
\hline 127 & Vanessa cardui (Linnaeus, 1758) & $\operatorname{Cos}$ & 1 & 1 & 1 & 1 & 1 & 1 & 1 & 1 & 1 & 1 \\
\hline 128 & Aglais io (Linnaeus, 1758) & ES & 1 & 1 & 1 & 1 & 1 & 1 & 1 & 1 & 1 & 1 \\
\hline 129 & Aglais urticae (Linnaeus, 1758) & ES & 1 & 1 & 1 & 1 & 1 & 1 & 1 & 1 & 1 & 1 \\
\hline 130 & Polygonia c-album (Linnaeus, 1758) & $\mathrm{ES}$ & 1 & 1 & 1 & 1 & 1 & 1 & 1 & 1 & 1 & 1 \\
\hline 131 & Polygonia egea (Cramer, 1775) & EO & 0 & 1 & 0 & 1 & 0 & 0 & 0 & 0 & 0 & 1 \\
\hline 132 & Araschnia levana (Linnaeus, 1758) & ES & 1 & 0 & 1 & 1 & 1 & 1 & 1 & 0 & 1 & 1 \\
\hline 133 & Nymphalis antiopa (Linnaeus, 1758) & Hol & 1 & 1 & 1 & 1 & 1 & 1 & 1 & 1 & 1 & 1 \\
\hline 134 & Nymphalis polychloros (Linnaeus, 1758) & EO & 1 & 1 & 1 & 1 & 1 & 1 & 1 & 1 & 1 & 1 \\
\hline 135 & Nymphalis xanthomelas (Esper, [1781]) & ES & 0 & 1 & 0 & 1 & 0 & 1 & 1 & 0 & 0 & 0 \\
\hline 136 & Nymphalis l-album (Esper, 1780) & $\mathrm{ES}$ & 0 & 1 & 0 & 1 & 0 & 1 & 1 & 0 & 0 & 0 \\
\hline 137 & Euphydryas maturna (Linnaeus, 1758) & $\mathrm{ES}$ & 0 & 1 & 0 & 0 & 0 & 0 & 1 & 1 & 0 & 1 \\
\hline 138 & Euphydryas aurinia (Rottemburg, 1775) & ES & 0 & 0 & 0 & 0 & 0 & 0 & 1 & 0 & 0 & 0 \\
\hline 139 & Melitaea cinxia (Linnaeus, 1758) & ES & 1 & 1 & 1 & 1 & 1 & 1 & 1 & 0 & 1 & 1 \\
\hline 140 & Melitaea phoebe (Goeze, 1779) complex & ES & 1 & 1 & 1 & 1 & 0 & 1 & 1 & 1 & 1 & 1 \\
\hline 141 & Melitaea arduinna (Esper, 1784) & EO & 0 & 0 & 1 & 1 & 1 & 0 & 0 & 0 & 0 & 0 \\
\hline 142 & Melitaea trivia (Schiffermüller, 1775) & EO & 0 & 1 & 1 & 1 & 1 & 1 & 1 & 1 & 1 & 0 \\
\hline 143 & Melitaea didyma (Esper, 1779) & ES & 1 & 0 & 1 & 1 & 1 & 1 & 1 & 1 & 1 & 1 \\
\hline 144 & Melitaea diamina (Lang, 1789) & ES & 0 & 1 & 0 & 1 & 0 & 1 & 1 & 1 & 0 & 0 \\
\hline 145 & Melitaea aurelia (Nickerl, 1850) & $\mathrm{EO}$ & 1 & 1 & 1 & 1 & 1 & 1 & 1 & 0 & 0 & 0 \\
\hline 146 & Melitaea athalia (Rottemburg, 1775) & ES & 1 & 1 & 1 & 1 & 1 & 1 & 1 & 1 & 1 & 1 \\
\hline 147 & Melitaea ornata (Christoph, 1893) & ES & 0 & 0 & 0 & 1 & 0 & 0 & 0 & 0 & 0 & 0 \\
\hline 148 & Limenitis populi (Linnaeus, 1758) & ES & 1 & 1 & 1 & 1 & 1 & 0 & 1 & 1 & 1 & 1 \\
\hline 149 & Limenitis camilla (Linnaeus, 1764) & ES & 1 & 0 & 1 & 1 & 1 & 1 & 1 & 1 & 0 & 0 \\
\hline 150 & Limenitis reducta (Staudinger, 1901) & EO & 1 & 0 & 1 & 1 & 1 & 1 & 1 & 1 & 1 & 0 \\
\hline 151 & Neptis sappho (Pallas, 1771) & ES & 1 & 1 & 1 & 1 & 1 & 1 & 1 & 1 & 0 & 1 \\
\hline 152 & Neptis rivularis (Scopoli, 1763) & ES & 0 & 0 & 1 & 1 & 0 & 1 & 1 & 1 & 1 & 0 \\
\hline 153 & Apatura metis (Freyer, 1829) & ES & 1 & 0 & 0 & 0 & 0 & 0 & 0 & 0 & 0 & 0 \\
\hline 154 & Apatura ilia (Schiffermüller, 1775) & ES & 1 & 1 & 1 & 1 & 1 & 1 & 1 & 1 & 1 & 1 \\
\hline 155 & Apatura iris (Linnaeus, 1758) & ES & 1 & 1 & 1 & 1 & 1 & 1 & 1 & 1 & 1 & 1 \\
\hline 156 & Kirinia roxelana (Cramer, 1777$)$ & $\mathrm{EO}$ & 0 & 0 & 1 & 1 & 1 & 1 & 0 & 0 & 0 & 0 \\
\hline 157 & Kirinia climene (Esper, 1784) & EO & 0 & 0 & 1 & 1 & 1 & 0 & 0 & 0 & 0 & 0 \\
\hline 158 & Pararge aegeria (Linnaeus, 1758 ) & EO & 1 & 1 & 1 & 1 & 1 & 1 & 1 & 1 & 1 & 1 \\
\hline 159 & Lasiommata megera (Linnaeus, 1767) & EO & 1 & 1 & 1 & 1 & 1 & 1 & 1 & 1 & 1 & 1 \\
\hline 160 & Lasiommata petropolitana (Fabricius, 1787) & ES & 0 & 0 & 0 & 1 & 0 & 0 & 1 & 1 & 1 & 0 \\
\hline 161 & Lasiommata maera (Linnaeus, 1758) & ES & 1 & 1 & 1 & 1 & 1 & 1 & 1 & 1 & 1 & 1 \\
\hline 162 & Lopinga achine (Scopoli, 1763) & ES & 0 & 0 & 1 & 0 & 0 & 0 & 0 & 0 & 0 & 0 \\
\hline 163 & Coenonympha rhodopensis (Elwes, 1900) & Med & 0 & 0 & 0 & 1 & 1 & 0 & 1 & 1 & 1 & 0 \\
\hline 164 & Coenonympha arcania (Linnaeus, 1760) & EM & 1 & 1 & 1 & 1 & 1 & 1 & 1 & 1 & 1 & 1 \\
\hline 165 & Coenonympha glycerion (Borkhausen, 1788) & ES & 1 & 0 & 0 & 1 & 1 & 1 & 1 & 0 & 0 & 0 \\
\hline 166 & Coenonympha orientalis $(\mathrm{Rebel}, 1910)$ & Mon & 0 & 0 & 0 & 0 & 0 & 0 & 1 & 1 & 0 & 0 \\
\hline 167 & Coenonympha leander (Esper, 1784) & EO & 0 & 1 & 1 & 1 & 1 & 1 & 1 & 0 & 1 & 1 \\
\hline 168 & Coenonympha pamphilus (Linnaeus, 1758) & EO & 1 & 1 & 1 & 1 & 1 & 1 & 1 & 1 & 1 & 1 \\
\hline 169 & Pyronia tithonus (Linnaeus, 1771) & EM & 0 & 1 & 1 & 1 & 1 & 1 & 1 & 1 & 1 & 0 \\
\hline 170 & Aphantopus hyperantus (Linnaeus, 1758) & ES & 1 & 1 & 1 & 1 & 1 & 1 & 1 & 1 & 1 & 1 \\
\hline 171 & Maniola jurtina (Linnaeus, 1758) & ES & 1 & 1 & 1 & 1 & 1 & 1 & 1 & 1 & 1 & 1 \\
\hline 172 & Hyponephele lycaon (Kühn, 1774) & ES & 1 & 0 & 1 & 1 & 0 & 1 & 1 & 1 & 1 & 0 \\
\hline 173 & Hyponephele lupina (Costa, 1836) & ES & 0 & 0 & 0 & 0 & 0 & 1 & 0 & 0 & 1 & 0 \\
\hline 174 & Erebia ligea (Linnaeus, 1758) & ES & 0 & 1 & 1 & 1 & 0 & 0 & 1 & 1 & 1 & 0 \\
\hline 175 & Erebia euryale (Esper, 1805) & Mon & 0 & 0 & 0 & 1 & 0 & 0 & 1 & 1 & 1 & 0 \\
\hline 176 & Erebia manto (Schiffermüller, 1775) & Mon & 0 & 0 & 0 & 0 & 0 & 0 & 0 & 1 & 0 & 0 \\
\hline 177 & Erebia epiphron (Knoch, 1783) & Mon & 0 & 0 & 0 & 0 & 0 & 0 & 0 & 1 & 1 & 0 \\
\hline 178 & Erebia orientalis (Elwes, 1909) & Mon & 0 & 0 & 0 & 1 & 0 & 0 & 0 & 0 & 0 & 0 \\
\hline 179 & Erebia aethiops (Esper, 1777) & EO & 0 & 0 & 1 & 1 & 0 & 0 & 1 & 1 & 0 & 0 \\
\hline 180 & Erebia medusa (Fabricius, 1787) & ES & 0 & 1 & 1 & 1 & 1 & 1 & 1 & 1 & 1 & 1 \\
\hline 181 & Erebia albergana (Prunner, 1798) & Mon & 0 & 0 & 0 & 1 & 0 & 0 & 0 & 0 & 0 & 0 \\
\hline 182 & Erebia gorge (Esper, 1805) & Mon & 0 & 0 & 0 & 0 & 0 & 0 & 0 & 0 & 1 & 0 \\
\hline 183 & Erebia rhodopensis (Nicholl, 1900) & Mon & 0 & 0 & 0 & 0 & 0 & 0 & 0 & 1 & 1 & 0 \\
\hline 184 & Erebia ottomana (Herrich-Schäffer, 1847) & Mon & 0 & 0 & 0 & 1 & 0 & 0 & 1 & 1 & 1 & 0 \\
\hline 185 & Erebia cassioides (Reiner \& Hochenwarth, 1792) & Mon & 0 & 0 & 0 & 0 & 0 & 0 & 0 & 1 & 1 & 0 \\
\hline 186 & Erebia pronoe (Esper, 1780$)$ & Mon & 0 & 0 & 0 & 0 & 0 & 0 & 0 & 1 & 1 & 0 \\
\hline 187 & Erebia melas (Herbst, 1796) & Mon & 0 & 0 & 0 & 0 & 0 & 0 & 0 & 1 & 1 & 0 \\
\hline
\end{tabular}




\begin{tabular}{|c|c|c|c|c|c|c|c|c|c|c|c|c|}
\hline 188 & Erebia oeme (Hübner, 1804) & Mon & 0 & 0 & 0 & 1 & 0 & 0 & 0 & 1 & 1 & 0 \\
\hline 189 & Erebia pandrose (Borkhausen, 1788) & $\mathrm{BM}$ & 0 & 0 & 0 & 0 & 0 & 0 & 0 & 1 & 1 & 0 \\
\hline 190 & Melanargia galathea (Linnaeus, 1758) & EO & 1 & 1 & 1 & 1 & 1 & 1 & 1 & 1 & 1 & 1 \\
\hline 191 & Melanargia larissa (Esper, 1784) & EO & 0 & 0 & 1 & 0 & 0 & 0 & 1 & 0 & 1 & 1 \\
\hline 192 & Satyrus ferula (Fabricius, 1793) & EO & 0 & 0 & 0 & 1 & 0 & 0 & 1 & 1 & 0 & 0 \\
\hline 193 & Minois dryas (Scopoli, 1763) & ES & 1 & 0 & 1 & 1 & 1 & 1 & 1 & 1 & 0 & 0 \\
\hline 194 & Hipparchia fagi (Scopoli, 1763) & EM & 0 & 1 & 0 & 1 & 0 & 1 & 1 & 0 & 1 & 0 \\
\hline 195 & Hipparchia syriaca (Staudinger, 1871) & EO & 0 & 0 & 0 & 0 & 0 & 1 & 1 & 0 & 0 & 0 \\
\hline 196 & Hipparchia semele (Linnaeus, 1758) & EM & 1 & 1 & 1 & 1 & 0 & 0 & 0 & 0 & 0 & 1 \\
\hline 197 & $\begin{array}{l}\text { Hipparchia volgensis (Mazochin- } \\
\text { Porshnjakov, 1952) }\end{array}$ & EM & 1 & 0 & 1 & 1 & 1 & 1 & 1 & 1 & 1 & 0 \\
\hline 198 & Hipparchia statilinus (Hufnagel, 1766) & EM & 1 & 0 & 0 & 1 & 0 & 1 & 1 & 1 & 1 & 1 \\
\hline 199 & Arethusana arethusa (Schiffermüller, 1775) & EO & 0 & 1 & 1 & 1 & 1 & 1 & 1 & 1 & 0 & 1 \\
\hline 200 & Brintesia circe (Fabricius, 1775) & EO & 1 & 1 & 1 & 1 & 1 & 1 & 1 & 1 & 0 & 1 \\
\hline 201 & Chazara briseis (Linnaeus, 1764) & $\mathrm{ES}$ & 1 & 0 & 1 & 1 & 0 & 1 & 1 & 1 & 1 & 0 \\
\hline
\end{tabular}

Following procedure summarizes results of faunal origin analyses, which is shown in Tab. 3 and Fig. 1.

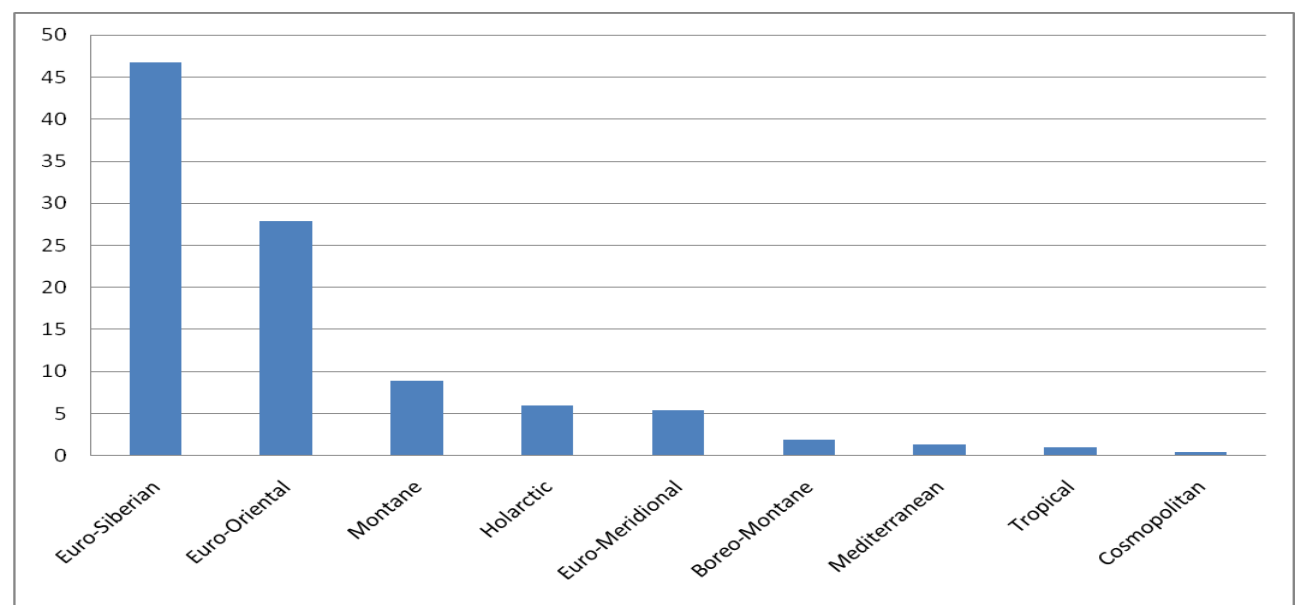

Fig. 1. Faunistic composition of Serbian butterflies.

Table. 3. Faunistic composition of Serbian butterflies: ES - Euro-Siberian, EO - Euro-Oriental, Mon Montane, Hol - Holarctic, EM - Euro-Meridional, BM - Boreo-Montane, Med - Mediterranean, Tro Tropical, Cos - Cosmopolitan.

\begin{tabular}{|l|c|c|c|c|c|c|c|c|c|}
\hline & \multicolumn{9}{|l|}{ The assemblages of species ("faunal elements") of Serbian butterflies } \\
\cline { 2 - 11 } & ES & EO & Mon & Hol & EM & BM & Med & Tro & Cos \\
\hline No. & 94 & 56 & 18 & 12 & 11 & 4 & 3 & 2 & 1 \\
\hline$\%$ & 46.76 & 27.86 & 8.95 & 5.97 & 5.47 & 1.9 & 1.4 & 1 & 0.5 \\
\hline
\end{tabular}

Results show that 94 (46\%) out of 201 established butterfly species belong to Euro-Siberian faunal elements. Besides them Euro-Oriental elements stand out with $56(27 \%)$ species. The other seven groups of faunal elements together make up only1/4 species in butterfly fauna of Serbia.

Further procedure analyses degree of closiness/difference between 11 appointed regions, comparing number of common species (Tab. 4). The results show that list number of common species in
Pannonicum and Dacicum area compared to other areas. This is understandable because it is about fauna of plains, with modified steppa character versus hillmountain fauna areas with primary forest vegetation. Large mountain ranges of Serbia have the largest number of common species, resulting in greatest number of habitats. Additionaly the value has been enlarged by dominant Euro-Siberian species as most represented one in that area. 
Table. 4. An overview of a total number of butterfly species in the studied areas (A) and the number of species common to both faunas (C).

\begin{tabular}{|c|c|c|c|c|c|c|c|c|c|c|}
\hline & \multirow[b]{2}{*}{ 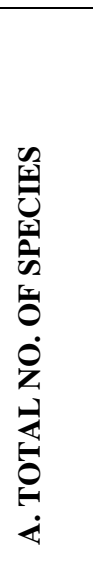 } & \multicolumn{9}{|c|}{ C } \\
\hline & & 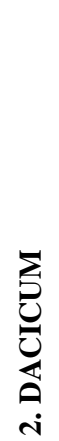 & 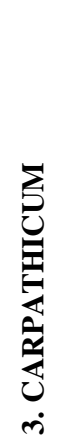 & 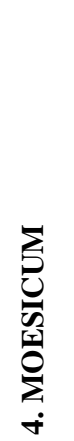 & 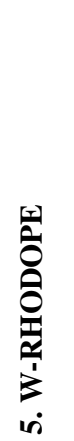 & 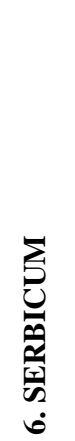 & 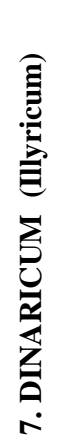 & 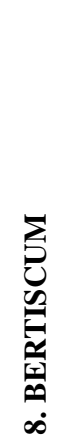 & 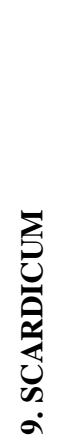 & 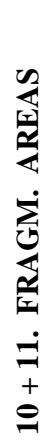 \\
\hline 1. PANNONICUM & 102 & 70 & 85 & 94 & 74 & 88 & 98 & 79 & 78 & 68 \\
\hline 2. DACICUM & 99 & & 81 & 92 & 78 & 87 & 89 & 77 & 83 & 73 \\
\hline 3. CARPATHICUM & 124 & & & 119 & 92 & 102 & 112 & 96 & 101 & 77 \\
\hline 4. MOESICUM & 159 & & & & 108 & 127 & 139 & 114 & 119 & 81 \\
\hline 5. W-RHODOPE & 111 & & & & & 95 & 98 & 85 & 92 & 68 \\
\hline 6. SERBICUM & 135 & & & & & & 126 & 102 & 107 & 76 \\
\hline 7. DINARICUM (Illyricum) & 156 & & & & & & & 113 & 124 & 85 \\
\hline 8. BERTISCUM & 134 & & & & & & & & 113 & 76 \\
\hline 9. SCARDICUM & 144 & & & & & & & & & 78 \\
\hline 10+11. FRAGMENT. AREAS & 97 & & & & & & & & & - \\
\hline
\end{tabular}

Table. 5. The degree of similarity coefficient $(\%)$ of the butterfly fauna in the analyzed areas obtained by Jaccard's coefficient.

\begin{tabular}{|c|c|c|c|c|c|c|c|c|c|}
\hline & 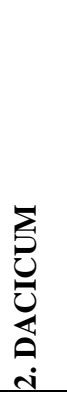 & 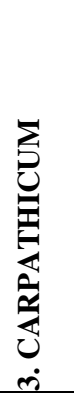 & 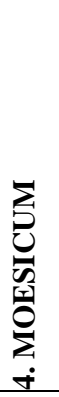 & 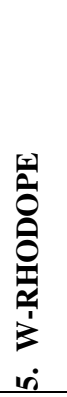 & 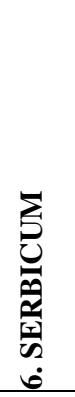 & 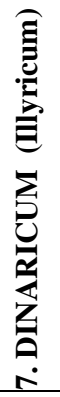 & 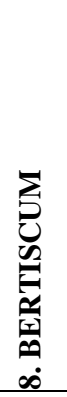 & 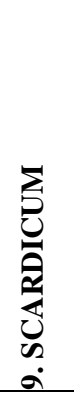 & 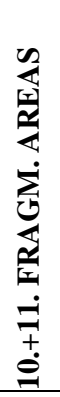 \\
\hline 1. PANNONICUM & 53 & 60 & 56 & 53 & 59 & 61 & 50 & 46 & 52 \\
\hline 2. DACICUM & & 57 & 55 & 59 & 59 & 53 & 49 & 52 & 59 \\
\hline 3. CARPATHICUM & & & 72 & 64 & 65 & 67 & 59 & 60 & 53 \\
\hline 4. MOESICUM & & & & 67 & 76 & 79 & 64 & 65 & 46 \\
\hline 5. W-RHODOPE & & & & & 63 & 58 & 53 & 56 & 48 \\
\hline 6. SERBICUM & & & & & & 76 & 61 & 62 & 49 \\
\hline 7. DINARICUM (Illyricum) & & & & & & & 64 & 70 & 50 \\
\hline 8. BERTISCUM & & & & & & & & 68 & 49 \\
\hline 9. SCARDICUM & & & & & & & & & 48 \\
\hline
\end{tabular}

Biology 


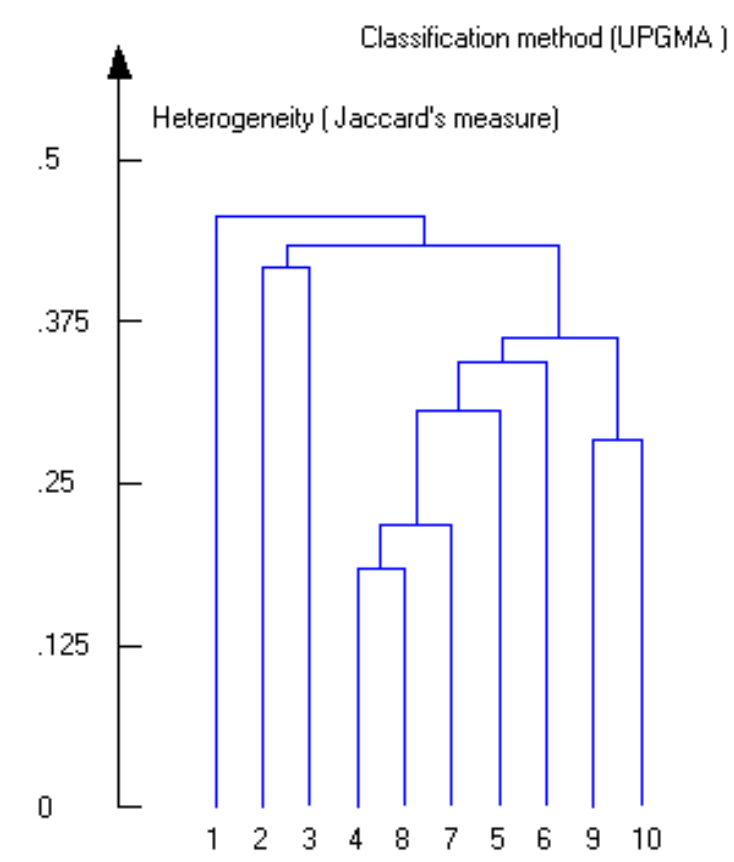

Fig. 2. Cluster tree of areas in Serbia according to composition of butterfly fauna.
Jaccard's coefficient analyses show the same value. The biggest difference exist between submediterranian and continental areas, as well as Pannonicum and Dacicum areas on one hand and inland on the other (Tab. 5).

In that sense Cluster analyses show clear confines within analyzed areas - four clusters. On the other hand, Cluster analyzes indicate mutual proximity of some regions within given Cluster (Fig. 2; Tab. 6). For example, it is completely normal for Pannonia region and Dacia area to be in the same Cluster, the same goes for Šar-Planina Mt. (Scardicum) area and Prokletije Mt. (Bertiscum).

As it is shown in Tab. 4 and Tab. 5 the exsistence of large number of common species in compared areas disables their zoogeographical separation. Actually, this fact indicates that analyzed area belongs to same zoogeographical unit of higher order. However, for zoogeographical separation into low order units, differential species can serve the purpose. These are the specific elements of fauna, usually presented in one area (Tab. 7).

Table. 6. Belonging to the clusters of selected regions in Serbia.

\begin{tabular}{|l|l|l|l|}
\hline Cluster 1 & Cluster 2 & Cluster 3 & Cluster 4 \\
\hline 1. Fragment. areas: & 2. Pannonicum & 4. Moesicum & 9. Bertiscum \\
10a. Sub-Aegean & 3. Dacicum & 5. Carpathicum & 10. Scardicum \\
10b. Sub-Adriaticum & & $\begin{array}{l}\text { 6. W-Rhodope } \\
\text { 7. Serbicum } \\
\text { 11. Ponišavlje }\end{array}$ & \\
& & 8. Dinaricum (Illyricum) & \\
\hline
\end{tabular}

Table. 7. Differential species (specific faunal elements) of certain regions.

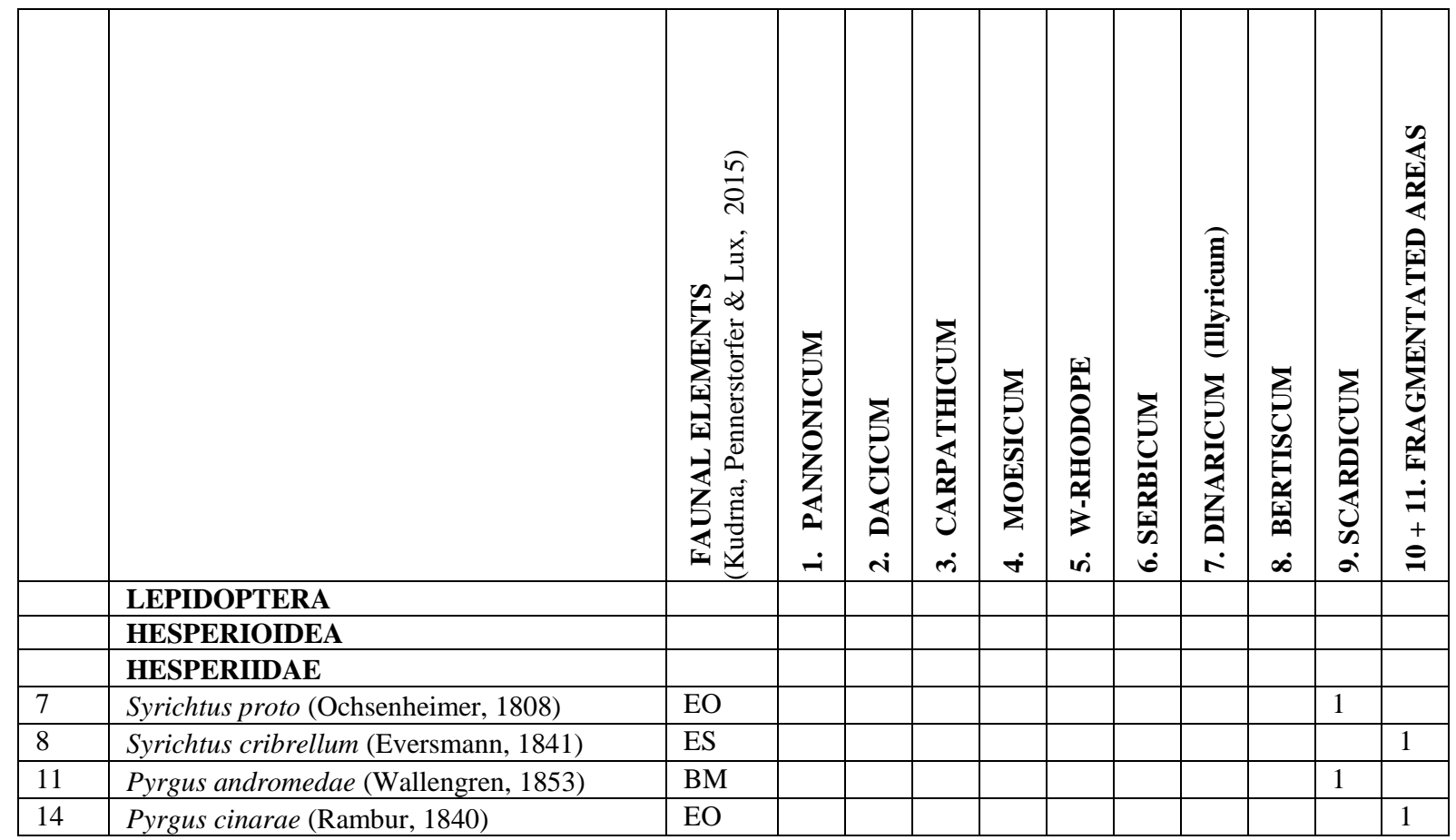

\section{Biology}




\begin{tabular}{|c|c|c|c|c|c|c|c|c|c|c|c|c|}
\hline & PAPILIONOIDEA & & & & & & & & & & & \\
\hline & PIERIDAE & & & & & & & & & & & \\
\hline 33 & Leptidea duponcheli (Staudinger, 1871) & EO & & & & & 1 & 1 & & 1 & & 1 \\
\hline 34 & Leptidea morsei (Fenton, 1882) & ES & 1 & & & & & & & & & \\
\hline 36 & Anthocharis gruneri Herrich-Schäffer, 1851 & EO & & & & & & & & & & 1 \\
\hline 37 & Euchloe ausonia (Hübner, 1806) complex & Med & 1 & 1 & & & & & & & & 1 \\
\hline 48 & Colias myrmidone (Esper, 1781) & EO & 1 & 1 & 1 & & & & & & & \\
\hline \multirow[t]{2}{*}{49} & Colias balcanica Rebel, 1903 & Mon & & & & & & & 1 & & & \\
\hline & LYCAENIDAE & & & & & & & & & & & \\
\hline 55 & Lycaena helle (Schiffermüller, 1775) & ES & & & & 1 & & & & & & \\
\hline 80 & Pseudophilotes bavius (Eversmann, 1832) & EM & & & & & & & & 1 & 1 & \\
\hline 85 & Phengaris teleius (Bergsträsser, 1779) & ES & 1 & & & & & & & & & \\
\hline 91 & Agriades optilete (Knoch, 1781) & $\mathrm{BM}$ & & & & & & & & & 1 & \\
\hline 97 & Polyommatus escheri (Hübner, 1823) & EM & & & & & & & 1 & & 1 & \\
\hline 102 & Polyommatus eros (Ochsenheimer, 1808) & ES & & & 1 & 1 & & & 1 & & 1 & \\
\hline \multirow[t]{2}{*}{108} & Polyommatus damon (Schiffermüller, 1775) & ES & & & & & & & & 1 & 1 & 1 \\
\hline & NYMPHALIDAE & & & & & & & & & & & \\
\hline 116 & Brenthis ino (Rottemburg, 1775) & ES & & & & 1 & & & & & & \\
\hline 119 & Boloria eunomia (Esper, 1799) & Hol & & & 1 & 1 & 1 & & & & & \\
\hline 121 & Boloria titania $($ Esper, 1793$)$ & Hol & & & & & & & 1 & 1 & 1 & \\
\hline 122 & Boloria selene (Schiffermüller, 1775) & Hol & & & 1 & 1 & & & 1 & & & \\
\hline 124 & Boloria pales (Schiffermüller, 1775) & Mon & & & & & & & & 1 & 1 & \\
\hline 125 & Boloria graeca (Staudinger, 1870) & Mon & & & & & & & & 1 & 1 & \\
\hline 131 & Polygonia egea (Cramer, 1775) & EO & & 1 & & 1 & & & & & & 1 \\
\hline 138 & Euphydryas aurinia (Rottemburg, 1775) & ES & & & & & & & 1 & & & \\
\hline 141 & Melitaea arduinna (Esper, 1784) & EO & & & 1 & 1 & 1 & & & & & \\
\hline 153 & Apatura metis (Freyer, 1829) & ES & 1 & & & & & & & & & \\
\hline 157 & Kirinia climene (Esper, 1784) & EO & & & 1 & 1 & 1 & & & & & \\
\hline 162 & Lopinga achine (Scopoli, 1763) & ES & & & 1 & & & & & & & \\
\hline 166 & Coenonympha orientalis (Rebel, 1910) & Mon & & & & & & & 1 & 1 & & \\
\hline 173 & Hyponephele lupina (Costa, 1836) & ES & & & & & & 1 & & & 1 & \\
\hline 176 & Erebia manto (Schiffermüller, 1775) & Mon & & & & & & & & 1 & & \\
\hline 177 & Erebia epiphron (Knoch, 1783) & Mon & & & & & & & & 1 & 1 & \\
\hline 178 & Erebia orientalis (Elwes, 1909) & Mon & & & & 1 & & & & & & \\
\hline 181 & Erebia albergana (Prunner, 1798) & Mon & & & & 1 & & & & & & \\
\hline 182 & Erebia gorge (Esper, 1805) & Mon & & & & & & & & & 1 & \\
\hline 183 & Erebia rhodopensis (Nicholl, 1900) & Mon & & & & & & & & 1 & 1 & \\
\hline 184 & Erebia ottomana (Herrich-Schäffer, 1847) & Mon & & & & 1 & & & 1 & 1 & 1 & \\
\hline 185 & Erebia cassioides (Reiner \& Hochenwarth, 1792) & Mon & & & & & & & & 1 & 1 & \\
\hline 186 & Erebia pronoe (Esper, 1780$)$ & Mon & & & & & & & & 1 & 1 & \\
\hline 187 & Erebia melas (Herbst, 1796) & Mon & & & & & & & & 1 & 1 & \\
\hline 188 & Erebia oeme (Hübner, 1804) & Mon & & & & 1 & & & & 1 & 1 & \\
\hline 189 & Erebia pandrose (Borkhausen, 1788) & $\mathrm{BM}$ & & & & & & & & 1 & 1 & \\
\hline 191 & Melanargia larissa (Esper, 1784) & EO & & & 1 & & & & 1 & & 1 & 1 \\
\hline 195 & Hipparchia syriaca (Staudinger, 1871) & EO & & & & & & 1 & 1 & & & \\
\hline 196 & Hipparchia semele (Linnaeus, 1758) & EM & 1 & 1 & 1 & 1 & & & & & & 1 \\
\hline
\end{tabular}

There are $46(23 \%)$ of these specific faunal elements. Their mapping can regionalize Serbia zoogeographicaly (Fig. 3).

The question of demarcation level mark remains open. Clasification principles and demarcation level defined in details by (Fosberg \& Pearsall, 1993). According to the modified Sclater-Wallace scheme of zoogeographical subdivision of land we have levels of Region-Subregion-Province.

Here separated entities could be identified as Province or Subprovince. In literature, the terms "Region", "District", "Area" etc. are also encountered for the same level of demarcation. 

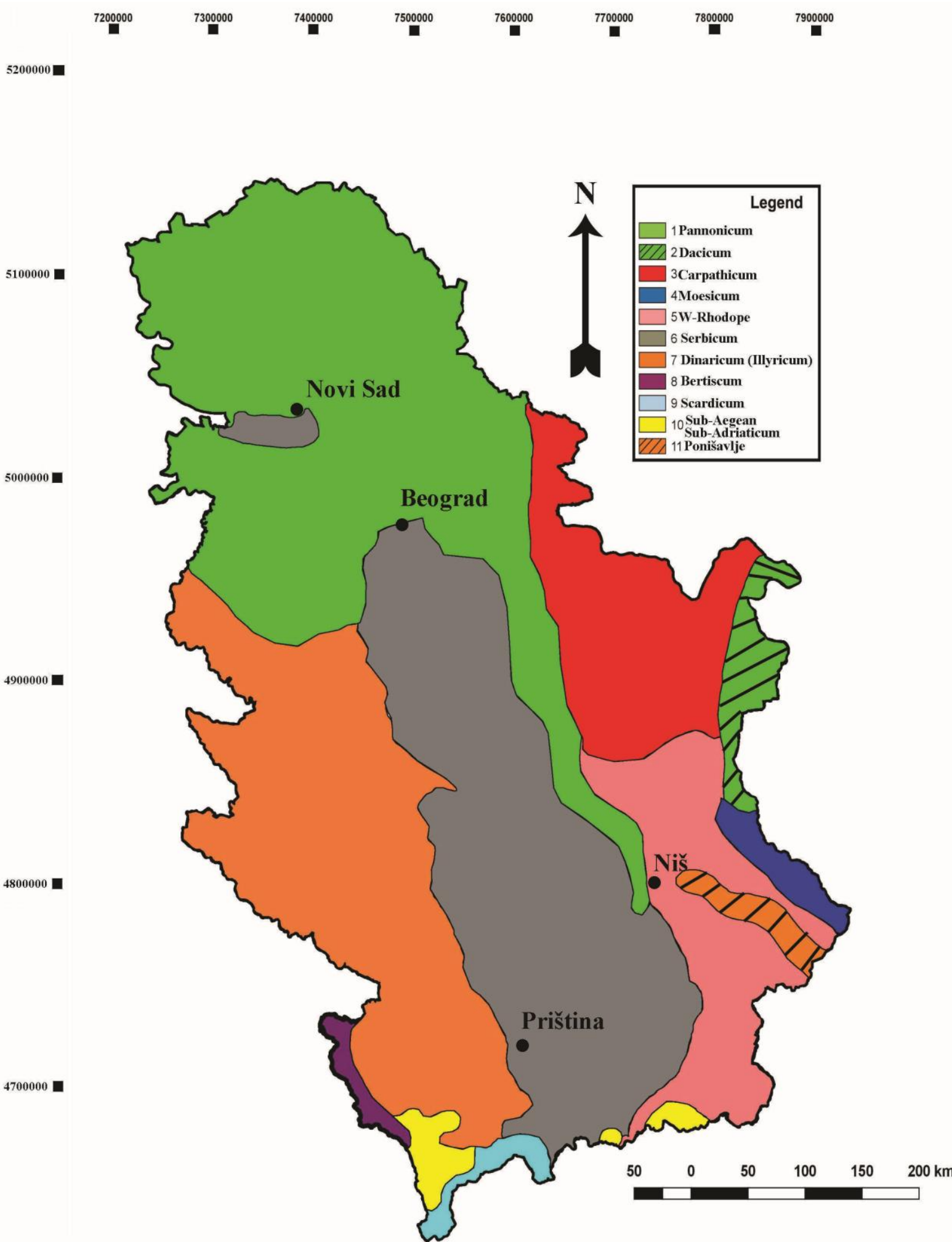

4600000 -

Fig. 3. Zoogeographical regionalization of the Serbia according to the affinity of local faunas of the butterflies (Lepidoptera: Hesperioidea \& Papilionoidea). See text below for further details. (Drawn by $\mathrm{PhD}$ AleksandarValjarević using GIS software's QGIS 2.14.2 and Global Mapper V 17.1. Data for map using by professor PredragJakšić, and after that georectified and digitized in Transverse Mercator projection and WGS 84 datum.) 


\section{Main characteristics of selected provinces:}

\section{The Pannonicum Province}

Geographically it extends to Pannonia and Peripannonia area comprising Vojvodina (except Fruška Gora Mt. and Vršac Mountains), Mačva and Morava Valley.

Fauna is poorer due to strong anthropogenic pressure.

Clima: Cfwb $\mathrm{x}^{\prime \prime}$, where $\mathrm{C}=$ mild temperatemesothermal climate; $\mathrm{f}=$ significant precipitation during all seasons; $\mathrm{w}=$ dry winters (in which the driest winter month average precipitation is less than one tenth the wettest summer month average precipitation); $\mathrm{b}=$ warmest month averaging below $22^{\circ} \mathrm{C}$ (but with at least 4 months averaging above $\left.10^{\circ} \mathrm{C}\right) ; \mathrm{x}^{\prime \prime}=$ the second precipitation maximum occurs in autumn

Natural Potential Vegetation: Genisto-Quercetum roboris s. lat.; Festucion rupicolae Aceri tatariciQuercetum.

Dominant biome type:

Biomes of submediterranean broad-leaved woodlands and schrubs and Biomes of steppes and woodland steppes.

Typical species of butterflies: Leptidea morsei (Fenton, 1882), Euchloe ausonia (Hübner, 1806), Colias erate (Esper, 1803), Colias myrmidone (Esper, 1781), Phengaris teleius (Bergsträsser, 1779), Apatura metis (Freyer, 1829), Hipparchia semele (Linnaeus, 1758).

\section{The Dacicum Province}

It includes parts of Vlaska lowland in Serbia, in other words area of former Dacian Sea in Timok region, Ključ, and Vidin region.

Fauna is exceptionally poorer due to strong anthropogenic pressure.

Clima: Cfwax", where $\mathrm{C}=$ mild temperatemesothermal climate; $\mathrm{f}=$ significant precipitation during all seasons; $\mathrm{w}=$ dry winters (in which the driest winter month average precipitation is less than one tenth the wettest summer month average precipitation); $\mathrm{a}=$ warmest month averaging above $22^{\circ} \mathrm{C} ; \mathrm{x}^{\prime \prime}=$ the second precipitation maximum occurs in autumn.

Natural Potential Vegetation: Quercetum pedunculiflorae moesiacum.

Dominant biome type:

Biomes of submediterranean broad-leaved woodlands and schrubs; Biomes of steppes and woodland steppes and Biomes of south-European mostly broad-leaved woodlands.

Typical species of butterflies: Euchloe ausonia (Hübner, 1806), Colias erate (Esper, 1803), Colias myrmidone (Esper, 1781), Polygonia egea (Cramer, 1775), Melanargia larissa (Esper, 1784), Hipparchia semele (Linnaeus, 1758).

\section{The Carpathicum Province}

Geographically it includes Vršac Mountains, Djerdap, Miroc, Homolje, Kucaj towards Rtanj Mt.

Clima: dominant Cfwbx", where $\mathrm{C}=$ mild temperate-mesothermal climate; $\mathrm{f}=$ significant precipitation during all seasons; $w=$ dry winters (in which the driest winter month average precipitation is less than one tenth the wettest summer month average precipitation) $; \mathrm{b}=$ warmest month averaging below $22^{\circ} \mathrm{C}$ (but with at least 4 months averaging above $\left.10^{\circ} \mathrm{C}\right) ; \mathrm{x}^{\prime \prime}=$ the second precipitation maximum occurs in autumn; as well as Dfwb $\mathrm{x}^{\prime \prime}$.

Natural Potential Vegetation: Fagetum montanum s. lat.

Dominant biome type:

Biomes of south-European mostly broad-leaved woodlands.

Typical species of butterflies: Colias myrmidone (Esper, 1781), Polyommatus eros (Ochsenheimer, 1808), Boloria eunomia (Esper, 1799), Boloria selene (Schiffermüller, 1775), Melitaea arduinna (Esper, 1784), Kirinia climene (Esper, 1784), Lopinga achine (Scopoli, 1763), Hipparchia semele (Linnaeus, 1758).

\section{The Moesicum Province}

Geographically it includes Stara Planina Mt.

Clima: Dfwbx", where D = continentalmicrothermal climate (a mean temperature above $10^{\circ} \mathrm{C}$ in the warmest months and a coldest month average below $\left.-3{ }^{\circ} \mathrm{C}\right) ; \mathrm{f}=$ significant precipitation during all seasons; $\mathrm{w}=$ dry winters (in which the driest winter month average precipitation is less than one tenth the wettest summer month average precipitation); $\mathrm{b}=$ warmest month averaging below $22^{\circ} \mathrm{C}$ (but with at least 4 months averaging above $\left.10^{\circ} \mathrm{C}\right) ; \mathrm{x}^{\prime \prime}=$ the second precipitation maximum occurs in autumn.

Natural Potential Vegetation: Abieti-Fagetum s. lat., Piceetum excelsae montanum s. lat., Piceetum excelsae subalpinum s. lat., Pinetum mugi s.lat.

Dominant biome type:

Biomes of European mostly coniferous forests of boreal type, some with elements of broad-leaved forests. 
Typical species of butterflies: Lycaena helle (Schiffermüller, 1775), Polyommatus eros (Ochsenheimer, 1808), Brenthis ino (Rottemburg, 1775), Boloria eunomia (Esper, 1799), Boloria selene (Schiffermüller, 1775), Polygonia egea (Cramer, 1775), Melitaea arduinna (Esper, 1784), Kirinia climene (Esper, 1784), Erebia orientalis Elwes, 1909, Erebia albergana (Prunner, 1798), Erebia ottomana (Herrich-Schäffer, 1847), Erebia oeme (Hübner, 1804), Hipparchia semele (Linnaeus, 1758).

\section{The W- Rhodope Province}

It includes Rhodope Mountains in Serbia, towards Rtanj Mt. in the North.

Clima: Dfwbx", where D = continentalmicrothermal climate (a mean temperature above $10^{\circ} \mathrm{C}$ in the warmest months and a coldest month average below $\left.-3^{\circ} \mathrm{C}\right) ; \mathrm{f}=$ significant precipitation during all seasons; $\mathrm{w}=$ dry winters (in which the driest winter month average precipitation is less than one tenth the wettest summer month average precipitation); $\mathrm{b}=$ warmest month averaging below $22^{\circ} \mathrm{C}$ (but with at least 4 months averaging above $\left.10^{\circ} \mathrm{C}\right) ; \mathrm{x}^{\prime \prime}=$ the second precipitation maximum occurs in autumn.

Natural Potential Vegetation: Quercetum frainettocerris s. lat., Fagetum montanum s. lat.

Dominant biome type:

Biomes of south-European mostly broad-leaved woodlands.

Typical species of butterflies: Leptidea duponcheli (Staudinger, 1871), Boloria eunomia (Esper, 1799), Melitaea arduinna (Esper, 1784), Kirinia climene (Esper, 1784). Also, Colias balcanica Rebel, 1903 can be expected in this area because it is distributed on the opposite side of border in Bulgaria

\section{The Serbicum Province}

It includes Šumadija and extends towards Skopska Crna Gora Mt. in the South; in the West it is bordered by River Ibar and extends towards Kopaonik Mt. and Rudnik Mt. Butterfly fauna of Fruska Gora Mt. also belongs to this area.

Clima: Cfwbx", where $\mathrm{C}=$ mild temperatemesothermal climate; $\mathrm{f}=$ significant precipitation during all seasons; $w=$ dry winters (in which the driest winter month average precipitation is less than one tenth the wettest summer month average precipitation); $\mathrm{b}=$ warmest month averaging below $22^{\circ} \mathrm{C}$ (but with at least 4 months averaging above $\left.10^{\circ} \mathrm{C}\right) ; \mathrm{x}^{\prime \prime}=$ the second precipitation maximum occurs in autumn.
Natural Potential Vegetation: Quercetum frainettocerris s.lat.

Dominant biome type:

Biomes of submediterranean broad-leaved woodlands and schrubs; Biomes of south-European mostly broad-leaved woodlands and Biomes of steppes and woodland steppes.

Typical species of butterflies: Leptidea duponcheli (Staudinger, 1871), Hyponephele lupina (Costa, 1836), Hipparchia syriaca (Staudinger, 1871).

\section{The Dinaricum (Illyricum) Province}

It covers an area from Cer Mt. in the North to Metohija Valley. In the East it extends to Kopaonik Mt. and towards border with Montenegro and Bosnia and Herzegovina in the West

Clima: Dfwbx", where $\mathrm{D}=$ continentalmicrothermal climate (a mean temperature above $10^{\circ} \mathrm{C}$ in the warmest months and a coldest month average below $\left.-3^{\circ} \mathrm{C}\right) ; \mathrm{f}=$ significant precipitation during all seasons; $\mathrm{w}=$ dry winters (in which the driest winter month average precipitation is less than one tenth the wettest summer month average precipitation); $\mathrm{b}=$ warmest month averaging below $22^{\circ} \mathrm{C}$ (but with at least 4 months averaging above $\left.10^{\circ} \mathrm{C}\right) ; \mathrm{x}^{\prime \prime}=$ the second precipitation maximum occurs in autumn.

Natural Potential Vegetation: Quercetum frainettocerris s. lat., Fagetum montanum s. lat. Piceetum excelsae montanum s. lat.

Dominant biome type:

Biomes of submediterranean broad-leaved woodlands and schrubs; Biomes of south-European mostly broad-leaved woodlands and Biomes of European mostly coniferous forests of boreal type.

Typical species of butterflies: Colias balcanica Rebel, 1903, Polyommatus escheri (Hübner, 1823), Polyommatus eros (Ochsenheimer, 1808), Boloria titania (Esper, 1793), Boloria selene (Schiffermüller, 1775), Euphydryas aurinia (Rottemburg, 1775), Coenonympha orientalis Rebel, 1910, Erebia ottomana (Herrich-Schäffer, 1847), Melanargia larissa (Esper, 1784), Hipparchia syriaca (Staudinger, 1871).

\section{The Bertiscum Province}

It includes Paštrik, Prokletije Mt. of Metohia, Bogićevica, Hajla, Žljeb and Mokra Gora Mt.

Clima: $\mathrm{ET}=$ polar and alpine climate with average temperatures below $10^{\circ} \mathrm{C}$ for all 12 months of the year. 
Natural Potential Vegetation: Quercetum petraeae s. lat., Piceetum excelsae montanum s. lat., Pinetum heldreichii s. lat., Oxytropidion dinaricae, Seslerion comosae.

Dominant biome type:

Biomes of European mostly coniferous forests of boreal type.

Typical species of butterflies: Leptidea duponcheli (Staudinger, 1871), Pseudophilotes bavius (Eversmann, 1832), Polyommatus damon (Schiffermüller, 1775), Boloria titania (Esper, 1793), Boloria pales (Schiffermüller, 1775), Boloria graeca (Staudinger, 1870), Coenonympha orientalis Rebel, 1910, Erebia manto (Schiffermüller, 1775), Erebia epiphron (Knoch, 1783), Erebia rhodopensis Nicholl, 1900, Erebia ottomana (Herrich-Schäffer, 1847), Erebia cassioides (Reiner \& Hochenwarth, 1792), Erebia pronoe (Esper, 1780), Erebia melas (Herbst, 1796), Erebia oeme (Hübner, 1804), Erebia pandrose (Borkhausen, 1788)

\section{The Scardicum Province}

Geographically it covers Šar-Planina Mt., Ošljak Mt., Kodža-Balkan Mt., Rudoka Mt., Vraca Mt., Korab Mt. and Koritnik Mt.

Clima: $\mathrm{ET}=$ polar and alpine climate with average temperatures below $10^{\circ} \mathrm{C}$ for all 12 months of the year.

Natural Potential Vegetation: Abieti-Fagetum s. lat., Piceetum excelsae montanum s. lat., Pinetum peucis s. lat., Edraiantho-Seslerion, Seslerion comosae.

Dominant biome type:

Biomes of stony grounds, pastures and woody on stony and Biomes of European mostly coniferous forests of boreal type.

Typical species of butterflies: Syrichtus proto (Ochsenheimer, 1808), Pyrgus andromedae (Wallengren, 1853), Pseudophilotes bavius (Eversmann, 1832), Agriades optilete (Knoch, 1781), Polyommatus escheri (Hübner, 1823), Polyommatus eros (Ochsenheimer, 1808), Polyommatus damon (Schiffermüller, 1775), Boloria titania (Esper, [1793]), Boloria pales (Schiffermüller, 1775), Boloria graeca (Staudinger, 1870), Hyponephele lupina (Costa, 1836), Erebia epiphron (Knoch, 1783), Erebia gorge (Esper, 1805), Erebia rhodopensis Nicholl, 1900, Erebia ottomana (Herrich-Schäffer, 1847), Erebia cassioides (Reiner \& Hochenwarth, 1792), Erebia pronoe (Esper, 1780), Erebia melas (Herbst, 1796), Erebia oeme (Hübner, 1804), Erebia pandrose
(Borkhausen, 1788), Melanargia larissa (Esper, 1784).

\section{0a. The Sub-Aegean Province (fragments)}

It includes Serbian parts of Crna Reka River basin and Serbian part of River Pčinja.

Clima: Cfwbx", where $\mathrm{C}=$ mild temperatemesothermal climate; $\mathrm{f}=$ significant precipitation during all seasons; $w=$ dry winters (in which the driest winter month average precipitation is less than one tenth the wettest summer month average precipitation); $\mathrm{b}=$ warmest month averaging below $22^{\circ} \mathrm{C}$ (but with at least 4 months averaging above $\left.10^{\circ} \mathrm{C}\right) ; \mathrm{x}^{\prime \prime}=$ the second precipitation maximum occurs in autumn.

Natural Potential Vegetation: Querco-Carpinetum orientalis s. lat.

Dominant biome type:

Biomes of submediterranean broad-leaved woodlands and shrubs.

Typical species of butterflies: Pyrgus cinarae (Rambur, 1840), Anthocharis gruneri HerrichSchäffer, 1851, Euchloe ausonia (Hübner, 1806), Leptidea duponcheli (Staudinger, 1871), Polygonia egea (Cramer, 1775).

\section{0b.The Sub-Adriaticum Province (fragments)}

It includes the valley of Beli Drim River and associated tributaries: Prizrenska Bistrica River, Topluga River, and Erenik (Ribnik) River.

Clima: Cfwbx", where $\mathrm{C}=$ mild temperatemesothermal climate; $\mathrm{f}=$ significant precipitation during all seasons; $w=$ dry winters (in which the driest winter month average precipitation is less than one tenth the wettest summer month average precipitation); $\mathrm{b}=$ warmest month averaging below $22^{\circ} \mathrm{C}$ (but with at least 4 months averaging above $\left.10^{\circ} \mathrm{C}\right) ; \mathrm{x}^{\prime \prime}=$ the second precipitation maximum occurs in autumn.

Natural Potential Vegetation: Quercetum trojanae s. lat.

Dominant biome type:

Biomes of submediterranean broad-leaved woodlands and shrubs.

Typical species of butterflies: Zerynthia cerisy (Godart, 1822), Euchloe ausonia (Hübner, 1806), Leptidea duponcheli (Staudinger, 1871), Polyommatus damon (Schiffermüller, 1775), Polygonia egea (Cramer, 1775) 


\section{The Ponišavlje Province}

It covers valleys of Nišava River, Visočica River, Jerma River and associated tributaries including southern slopes of Stara Planina Mt. and Vidlič Mt.

Clima: Cfwbx", where $\mathrm{C}=$ mild temperatemesothermal climate; $\mathrm{f}=$ significant precipitation during all seasons; $\mathrm{w}=$ dry winters (in which the driest winter month average precipitation is less than one tenth the wettest summer month average precipitation); $\mathrm{b}=$ warmest month averaging below $22^{\circ} \mathrm{C}$ (but with at least 4 months averaging above $\left.10^{\circ} \mathrm{C}\right) ; \mathrm{x}^{\prime \prime}=$ the second precipitation maximum occurs in autumn.

Natural Potential Vegetation: Syringo-Carpinetum orientalis, Quercetum Frainetto-cerris s. lat.

Dominant biome type:

Biomes of submediterranean broad-leaved woodlands and shrubs.

Typical species of butterflies: Syrichtus cribrellum (Eversmann, 1841), Leptidea duponcheli (Staudinger, 1871), Polyommatus damon (Schiffermüller, 1775).

\section{DISCUSSION AND CONCLUSIONS}

Although in general it belongs to Central European faunal type, butterfly fauna of Serbia could still be zoogeographicaly separated. This separation is enabled by specific geological and tectonic history, diversifying ecological condition in territory, by richness and presence of specific faunal elements. Phytogeographical separation of Serbia (Gajić, 1984) identifies four main provinces: Pannonia, Moesia, Illiricum and Skardicum-Pind. (Hadži, 1931) geographical chart have three entities separated: Pannonia, Moesia and Illiricum. In this paper, presented chart basically coincides with Gajić and Hadži with one difference, that 11 zoogeographical entities have been recognized.

\section{ACKNOWLEDGEMENTS}

I am grateful to Dr Ana Savić, University of Niš, Faculty of Sciences; Dr Dragana Ranđelović, University of Belgrade, Faculty of Mining and Geology (Department for Mineralogy, Crystallography, Petrology and Geochemistry) and to Dr Aleksandar Valjarević, University of Priština (Kosovska Mitrovica), Faculty of Sciences, for their valuable assistance and useful advices.

\section{REFERENCES WITH ANNOTATED BIBLIOGRAPHY}

Adamović, L. 1909. Die Vegetationsverhältnisse der Balkanländer. In A. Engler\& O. Drude Eds., Die Vegetation der Erde.Leipzig. Band XI.

Andjus, Lj. 2008. Butterflies (Lepidoptera, Hesperioidea and Papilionoidea) from the collection of the Natural History Museum in Belgrade. Natural History Museum in Belgrade, Belgrade, 40, pp. 194.

Carnelutti, J. 1981. Horološka, ekološka in zoogeografska analiza makrolepidopterov slovenskega ozemlja.Ljubljana.

Dennis, R.L.H., Williams, W., \& Shreeve, T.G. 1998. Faunal structures among European butterflies: Evolutionary implications of bias for geography, endemism and taxonomic affiliation. Ecography, 21(2), pp. 181-203. doi:10.1111/j.1600-0587.1998.tb00672.x

Dodok, I. 2003. Dnevni leptiri (Lepidoptera: Hesperioidea I Papilionoidea) klisure reke Đetinje u zapadnoj Srbiji / The Butterflies fauna (Lepidoptera: Hesperioidea \& Papilionoidea) in the Gorge of the Djetinja River in West Serbia.Zaštita prirode, 54(12), pp. 89-105.

Đurić, M., \& Franeta, F. 2011. First study of the butterflies (Lepidoptera: Papilionoidea) of Mt. Mučanj. Acta entomologica serbica, 16(1/2), pp. 8190.

Fosberg, F.R., \& Pearsall, H.S. 1993. Clasification of non-marine ecosystems. Atoll Research Bulletin, 389, pp. 1-38.

Fukarek, P., Jovanović, B., \& eds., 1983. Karta prirodne potencijalne vegetacije SFR Jugoslavije 1: 1. 000. 000.Skopje: Naučno veće vegetacijske karte Jugoslavije, Šumarski fakultet.

Gajić, M. 1984. Florni elementi Srbije. In M. Janković\& et al. Eds., Vegetacija SR Srbije I..Beograd: SANU., pp. 317-397.

Gross, F.J. 1961. Zur Evolution euro-asiatischer Lepidopteren. In Verhandlungen der Deutschen Zoologischen Gesellschaft.Saarbrücken., pp. 461477.

Gradojević, M. 1930. Leptirovi Srbije - Diurna / Les papillons de Serbie I. Diurna. Glasnik Jugoslovenskog entomoloskog društva, Beograd, 56(1-2), pp. 133-158.

Hadži, J. 1931. Zoogeografska karta Kraljevine Jugoslavije.Beograd: Zbirka karata Geografskog Društva.

Hormuzaki, C. 1929. Grundlinien für die Biogeographie und Biogenese der Europäischen Makrolepidopteren.Buletinul Facultati de stinte din Cernauti, 3, pp. 111-159.

Hormuzaki, C. 1930. Die Entwicklung der europäischen Lepidopterenfauna seit der Tertiärzeit im Lichte der Paläoggeographie, Pflanzengeographie und Paläontologie. Buletinul Facultati de stinte din Cernauti, 4, pp. 50-135.

Ilešić, S. 1961. Geografska regionalizacija Jugoslavije / Sur les problèmes de la délimitation et classification des régions géographiques d'après 
l'example de la Yougoslavie. Geografski vestnik, Ljubljana, 33, pp. 3-25.

Jaccard, P. 1902. Lois de distribution florale dans la zone alpine. Bull. Soc. Vaudoise Sci. Nat., 38, pp. 69-130.

Jakšić, P. 1979. Dnevni leptiri SAP Kosovo.Priština., pp. 1-150.

Jakšić, P. 1986. Specifični elementi faune Lepidoptera nekih kosovskih klisura. Priroda Kosova. Priština, 6 , pp. 93-107.

Jakšić, P. 1998. Altitudinal distribution and biogeographical division of the butterflies of Balkan Peninsula (Lepidoptera Hesperioidea \& Papilionoidea). University Thought, 5(2), pp. 77-88.

Jakšić, P. 1998. Dnevni leptiri (Lepidoptera: Hesperioidea \& Papilionoidea) Šar-planine / The Butterflies of the Šar-planina Mt. (Lepidoptera: Hesperioidea \& Papilionoidea). Zaštita prirode, Beograd, 50, pp. 229-252.

Jakšić, P., \& Ristić, G. 1999. New and rare species of Lepidoptera in Yugoslavia. Acta entomologica serbica, 4(1/2), pp. 63-74.

Jakšić, P. 2003. Dnevni leptiri (Lepidoptera: Hesperioidea and Papilionoidea) Suve planine. Zaštita prirode, Beograd,54(1-2), pp. 71-87.

Jakšić, P. 2003. Fauna leptira (Lepidoptera: Zygaenidae, Hesperioidea i Papilionoidea). In L. Amidžić, M.M. Janković, \& P. Jakšić Eds., Metohijske Prokletije - prirodna i kulturna baština / Prokletije of Metohija: The natural and cultural heritage.Beograd: Zavod za zaštitu prirode Srbije., pp. 234-261.

Jakšić, P. 2006. Pregled utvrđenih vrsta dnevnih leptira borskog područja (Lepidoptera: Hesperioidea I Papilionoidea). SANU, Zbornik radova o fauni Srbije, 7, pp. 35-86.

Jakšić, P., Verovnik, R., \& Dodok, I. 2008. Overview of Prime Butterfly Areas in Serbia/Pregled Odabranih područja za dnevne leptire u Srbiji. In P. Jakšić Ed., Prime Butterfly Areas in Serbia: A tool for nature conservation in Serbia / Odabrana područja za dnevne leptire - put za ostvarenje zaštite prirode u Srbiji.Beograd: Habiprot Ed., pp. 43-203.

Jakšić, P. 2011. Butterfly species (Lepidoptera: Hesperioidea \& Papilionoidea) new to the Serbian fauna. Biologica Nyssana, 2(1), pp. 45-50.

Jakšić, P., \& Nahirnić, A. 2011. Dnevni leptiri Zasavice. Službeni glasnik i Specijalni rezervat prirode "Zasavica", Beograd, , pp. 1-100.

Jakšić, P., \& Nahirnić, A. 2014. Vodič kroz faunu dnevnih leptira Kopaonika.Kopaonik: JP Nacionalni Park "Kopaonik"., pp. 1-183.

Jakšić, P. 2015. Aspects of butterfly zoogeography of some Pannonian island mountains. Matica Srpska J. Nat. Sci. Novi Sad., 128, pp. 7-19.

Jakšić, P. 2015. On the new and rare Lepidoptera in Serbian fauna. Matica Srpska J. Nat. Sci. Novi Sad., 129, pp. 73-83.

Karadžić, B., Marinković, S., 2009. Kvantitativna ekologija. - Institut za biološka istraživanja "Siniša Stanković", Beograd.
Kobelt, W. 1904. Iconographie der Land-und Süsswasser-Mollusken mit vorzüglicher berücksichtigung der Europaischen noch nicht abgebildeten Arten.Wiesbaden.

Kogovšek, N., Deželak, U., Gredar, T., Popović, M., Ramšak, B., \& Tratnik, N. 2012. Poročilo o delu skupine za dnevne metulje. In Zbornik Ekosistemi Balkana Srbija.Ljubljana: Društvo študentov biologije., pp. 21-28.

Kostrowicki, A.S. 1969. Geography of the Palaearctic Papilionoidea (Lepidoptera).Krakow: Panstwowe wydawnictwo naukowe.

Kudrna, O., Pennerstorfer, J., \& Lux, K. 2015. Distribution Atlas of European Butterflies and Skippers.Schwanfeld: Wissenschaftlicher Verlag Peks e. K.

de Lattin, G. 1967. Grundriss der Zoogeographie.Stuttgart: Gustav Fischer Verlag.

Lorković, Z., \& Siladjev, S. 1982. Der Erstfund der Raupe von Apatura metis (Freyer) in Europa und deren Biotop (Lep., Nymphalidae). Atalanta, 13(2), pp. 126-135.

Matvejev, S. 1968. Kopnena fauna Srbije sa faunističkom kartom. - Enciklopedija Jugoslavije, Srbija 7: pp. 659-667. Leksikografski zavod FNRJ, Zagreb.

Matvejev, S. 1980. Faunistička karta Srbije. In Enciklopedija Jugoslavije.Zagreb: Jugoslavenski leksikografski zavod., p. 661. 7.

Matvejev, S.D., \& Puncer, I.J. 1989. Karta bioma predeli Jugoslavije i njihova zaštita.Beograd: Prirodnjački muzej u Beogradu.

Mihailović, D.T., Lalić, B., Drešković, N., Mimić, G., Djurdjević, V., \& Jančić, M. 2014. Climate change effects on crop yields in Serbia and related shifts of Köppen climate zones under the SRES-A1B and SRES-A2. Int. J. Climatol, November. doi:10.1002/joc.4209

Nahirnić, A. 2011. Supplement to butterfly fauna (Hesperioidea and Papilionoidea) of Grza River Gorge (Eastern Serbia). Biologica Nyssana, Niš, 2(2), pp. 107-117.

Nahirnić, A., Jakšić, P., \& Viborg, A.L. 2015. Colias caucasica balcanica (Pieridae) rediscovered in Montenegro, with additional new records for Serbia. Phegea, 43(1), pp. 6-10.

Pagenstecher, A. 1909. Die geographische Verbreitung der Schmetterlinge.Jena: Gustav Fischer Verlag.

Pavlović, S.P. 1912. Mekušci iz Srbije I. Suvozemni puževi.Beograd: Državna štamparija Kraljevine Srbije.

Popović, M., Radaković, M., Đurđević, A., Franeta, F., \& Verovnik, R. 2014. Distribution and threats of Phengaris teleius (Lepidoptera: Lycaenidae) in Northern Serbia. Acta Zoologica Academiae Scientiarum Hungaricae, 60(2), pp. 173-183.

Popović, M., Radevski, Đ., Miljević, M., \& Đurić, M. 2014. First record of Pyrgus cinarae (Lepidoptera: Hesperiidae) in Serbia. Acta entomologica 
serbica, 19(1/2), pp. 45-51. Serb. Summary, Figs. 13 , map 1.

Popović, M., \& Đurić, M. 2014. Dnevni leptiri Stare planine (Lepidoptera: Papilionoidea) / Butterflies of Stara Planina (Lepidoptera: Papilionoidea).Beograd: Javno preduzeće "Srbijašume"; Beograd: HabiProt, Str.., pp. 1-207.

Rebel, H. 1932. Zur Frage der europäischen Faunenelemente. Annalen des Naturhistorischen Museum in Wien, 46, pp. 49-55.

Sokal, R.R., \& Rohlf, F.J. 1995. Biometry, 3rd ed.New York: W.H. Freeman Co.

Stevanović, V., Jovanović, S., \& Lakušić, D. 1995. Diverzitet vegetacije Jugoslavije. In V. Stevanović\& V. Vasić Eds.,Biodiverzitet Jugoslavije sa pregledom vrsta od međunarodnog značaja.Beograd: Biološki fakultet i Ecolibri.

van Swaay, C., Jakšić, P., \& Đurić, M. 2007. Notes on some summer butterflies (Lepidoptera: Hesperioidea and Papilionoidea) of Eastern Serbia. Acta entomologica serbica, Beograd, 12(1), pp. 1-10.

Szabo, E. 2002. Biogeographical Analysis of the Butterfly Fauna of the Eastern and Southern Carpathians.Debrecen: University of Debrecen, Faculty of Science.

Szent-Ivanny, J. 1937. Sketch of the zoogeographical division of the Carpathians Basin regarding the distribution of the Macrolepidoptera. Annales Musei Nationalis Hungarici, Pars Zoologica, 31, pp. 129136.

Todorova, W., \& Petkoff, P. 1915. Beitrag zur Macrolepidopteren-Fauna der Umgebung von

\footnotetext{
*E-mail: jaksicpredrag@gmail.com
}

Tzaribrod und Trn.Sofia: Arbeiten der Bulgarischen Naturforschenden Gesellschaft., pp. 128-147.

Tuleschkow, K. 1932. Erster Beitrag zur Schmetterlingsfauna des Westbalkans (Westliche Stara-Planina).София: Трудоветь на ББлгарското Природоизпитателно Дружество., pp. 307-312. 15 16.

Varga, Z. 1977. Das Prinzip der areal analytischen Methode in der Zoogeographie und die Faunelemente-Einteilung der europäischen Tagschmetterlinge (Lepidoptera: Diurna). Acta biologica debrecina, Debrecin, 14, pp. 223-285.

Varga, Z. 2002. Zoologische Forschungen in den Hochgebirgen von Zentralasien: Erlebnisse und Ergebnisse.Humboldt Nachrichten, 21, pp. 3-53.

Warnecke, G. 1959. Verzeichnis der boreoalpinen Lepidopteren. Zitschrift der Wiener Entomologischen Geselschaft,44(2), pp. 17-26.

Wiltshire, E.P. 1945. Studies in the geography of Lepidoptera. III: The zoogeographical classification of West Palearctic species. The Entomologist, 78(987), pp. 113-116.

Zaragüeta-Bagils, R., Bourdon, E., Ung, V., VignesLebbe, R., \& Malécot, V. 2009. On the International Code of Area Nomenclature (ICAN). Journal of Biogeography, 36(8), pp. 1617-1619.

Zečević, M. 2002. Fauna leptira Timočke krajine (Istočna Srbija).Bor: Bakar; Zaječar: Narodni muzej.

Živojinović, S. 1950. Fauna insekata šumske domene Majdanpeka / Le Faune des Insectes du Domaine forestier de Majdanpek.Beograd: Srpska akademija nauka. Posebna izdanja, knjiga CLX, Institut za ekologiju i biogeografiju, knjiga 2 . 